\title{
Pd(II)-Catalyzed Acetoxylation of Uracil via Electrophilic Palladation
}

\author{
Hyun Seung Lee, Se Hee Kim, and Jae Nyoung Kim* \\ Department of Chemistry and Institute of Basic Science, Chonnam National University, Gwangju 500-757, Korea \\ *E-mail:kimjn@chonnam.ac.kr \\ Received November 12, 2009, Accepted November 26, 2009
}

Key Words: Palladium, Uracil, Acetoxylation, Electrophilic palladation

Palladium-catalyzed C-H activation/functionalization reactions are of great utility in organic chemistry because they enable the direct replacement of various $\mathrm{C}-\mathrm{H}$ bonds with new functionality. ${ }^{1}$ Among them a palladium-catalyzed oxidative functionalization of $\mathrm{C}-\mathrm{H}$ bonds has received much attention. Especially, a Pd-catalyzed acetoxylation or hydroxylation have received a special attention as one of the best oxidative functionalization of $\mathrm{C}-\mathrm{H}$ bond, ${ }^{2}$ and various substrates have been examined in these regards including benzylpyridine, ${ }^{2 a}$ arylpyridine, ${ }^{2 \mathrm{~b}, \mathrm{~h}}$ benzo[ $\left.h\right]$ quinoline, ${ }^{2 \mathrm{c}}$ and acetophenone oxime ether. ${ }^{2 \mathrm{~d}}$ Very recently, acetoxylation at the 3-position of indole moiety was published by Suna and co-workers. ${ }^{3}$ The nucleophilic nature of the carbon atom at the 5-position of uracil ring is very similar with that of the carbon atom at the 3-position of indole. In addition, 5-hydroxyuracil and related compounds have been known as important oxidative lesions of DNA. ${ }^{4}$ Thus, synthesis and applications of these compounds has received much attention. ${ }^{5}$ To the best of our knowledge, a palladium-catalyzed oxidative functionalization of uracil has not been reported. ${ }^{6}$ Thus we decided to examine the preparation of 5-acetoxyuracil derivatives via a Pd(II)-catalyzed direct acetoxylation of uracil moiety. $^{7}$

Initially, we examined the reaction of uracil (1a) under the conditions of $\mathrm{Pd}(\mathrm{OAc})_{2}(10 \mathrm{~mol} \%) / \mathrm{PhI}(\mathrm{OAc})_{2}(2.5$ equiv $)$ in

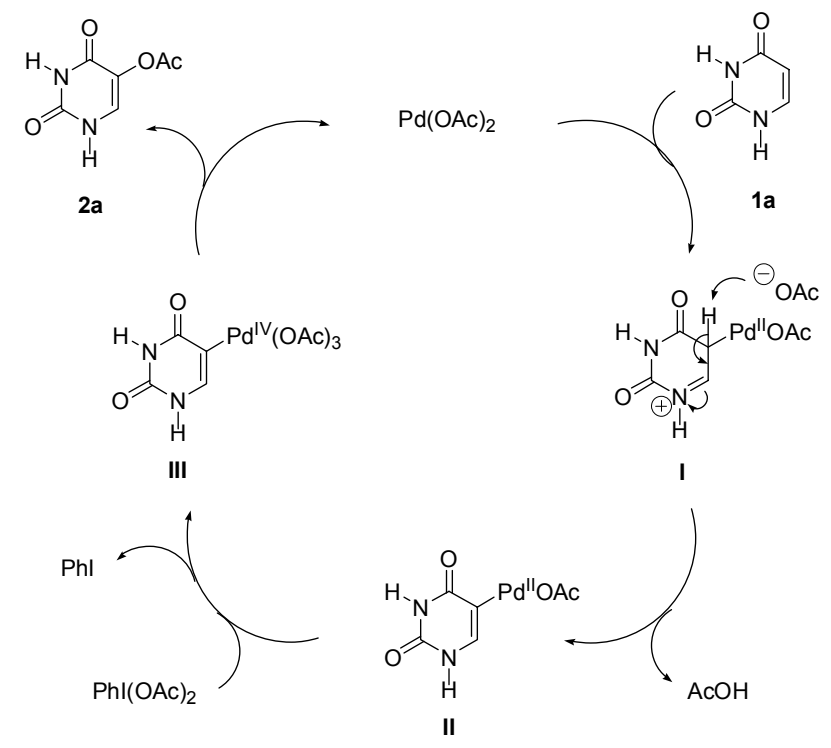

Scheme 1
AcOH $\left(100{ }^{\circ} \mathrm{C}, 2 \mathrm{~h}\right)$, and the desired product $2 \mathrm{a}$ was obtained in $68 \%$ yield (vide infra, conditions $\mathrm{B}$ ). The reaction proceeded at $60{ }^{\circ} \mathrm{C}(3 \mathrm{~h})$ more cleanly, and $\mathbf{2 a}$ was obtained in an increased yield ( $72 \%$, vide infra, conditions A). The plausible reaction mechanism is shown in Scheme 1: (i) an electrophilic palladation at the electron-rich 5-position of 1a to form 5-palladauracil intermediate (II) by liberation of acetic acid via the intermediate (I), (ii) oxidation of (II) to (III) by phenyliodonium diacetate (PIDA) with liberation of iodobenzene, as Sanford and coworkers already reported, ${ }^{1,2}$ and (iii) the final reductive removal of $\mathrm{Pd}$ (II) to produce 5-acetoxyuracil (2a). The reaction of $\mathbf{1 a}$ and PIDA at $60{ }^{\circ} \mathrm{C}$ without a palladium catalyst did not produce any trace amounts of $2 \mathrm{a}^{8}{ }^{8}$ The use of $\mathrm{Cu}(\mathrm{OAc})_{2}$, oxone, air, or $\mathrm{K}_{2} \mathrm{~S}_{2} \mathrm{O}_{8}$ as an oxidant instead of PIDA were ineffective. The reactions in DMF, $\mathrm{CH}_{3} \mathrm{CN}$, and 1,4-dioxane were also ineffective.

Encouraged by the results, we examined the reactions of various uracil derivatives $\mathbf{1 b} \mathbf{b} \mathbf{h}$, and the results are summarized in Table 1. As shown in Table 1, the reactions of 6-methyluracil (1b), 1,3-dimethyluracil (1c), and 1,3,6-trimethyluracil (1d) afforded the corresponding 5-acetoxy derivatives $\mathbf{2 b - d}$ in 56 $71 \%$ isolated yields (entries $2-4$ ). The yields of $\mathbf{2 b}$-d were somewhat lower $(45-55 \%)$ at $100{ }^{\circ} \mathrm{C}$ (conditions B) than those of the conditions $\mathrm{A}\left(60{ }^{\circ} \mathrm{C}\right)$ due to increased formation of intractable side products. The reactions of $\mathbf{1 e - h}$, however, were very sluggish at $60{ }^{\circ} \mathrm{C}$. But we obtained compounds $\mathbf{2 e - h}$ at $100{ }^{\circ} \mathrm{C}$ in low to moderate yields $(23-55 \%)$. It is interesting to note that triacetoxy derivatives $\mathbf{3 e}$ and $\mathbf{3 f}$ were formed together in variable yields for the benzyl-substituted uracil derivatives (entries 5 and 6). ${ }^{3,4 a}$ The plausible mechanism for the formation of triacetoxy compounds are suggested in Scheme 2. We could not isolate the corresponding triacetoxy compounds in appreciable amounts for other entries. In the reaction of $\mathbf{1 h}$, the yield of $\mathbf{2 h}$ was low and starting material $\mathbf{1 h}$ was recovered in $14 \%$. In addition, we isolated $16 \%$ of 5 -acetoxyuracil (2a) which resulted by the cleavage of $N$-glycoside bond under the acidic conditions. ${ }^{9}$ The reaction of 1,3,5-trimethyluracil (1i) failed, as expected due to the presence of 5-methyl group. The reaction of methyl 1,3-dimethylorotate (1j) also failed completely presumably due to the presence of electron-withdrawing ester group which diminish the nucleophilicity at the C-5 position of uracil ring.

In summary, a palladium-catalyzed electrophilic acetoxylation at the electron-rich 5-position of uracil was examined. The reaction proceeded via the electrophilic palladation of $\mathrm{Pd}(\mathrm{II})$ at the 5-position of uracil ring, and regeneration of $\mathrm{Pd}(\mathrm{II})$ was 


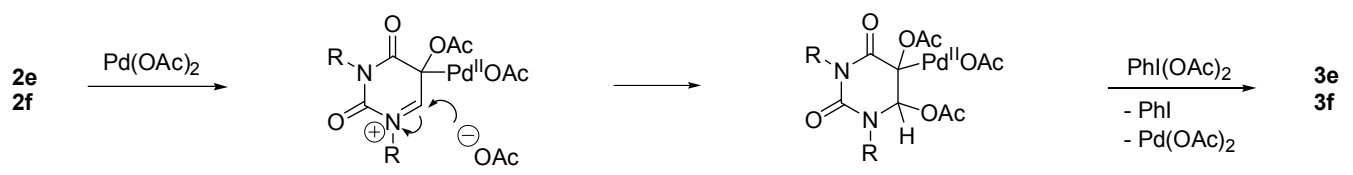

Scheme 2

Table 1. Pd(II)-catalyzed 5-acetoxylation of uracil derivatives

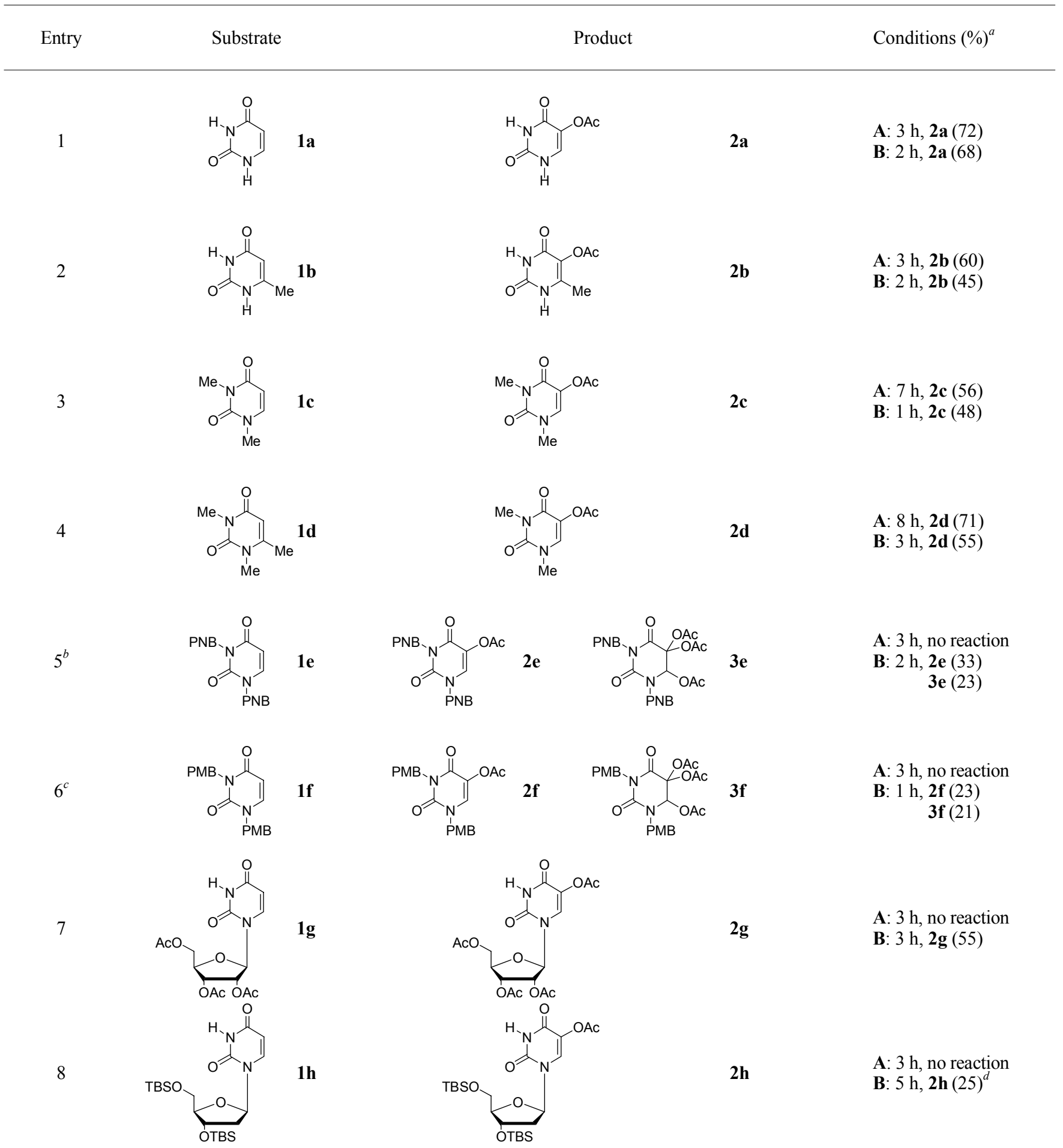

${ }^{a}$ Conditions: $\mathrm{Pd}(\mathrm{OAc})_{2}(10 \mathrm{~mol} \%), \mathrm{PhI}(\mathrm{OAc})_{2}\left(2.5\right.$ equiv), $\mathrm{AcOH}, \mathbf{A}: 60{ }^{\circ} \mathrm{C} ; \mathbf{B}: 100{ }^{\circ} \mathrm{C} .{ }^{b} \mathrm{PNB}$ is p-nitrobenzyl. ${ }^{c} \mathrm{PMB}$ is $p$-methoxybenzyl. ${ }^{d} \mathbf{1 h}(14 \%)$ and 2a (16\%) were isolated. 
effectively occurred with the aid of PIDA (phenyliodonium diacetate).

\section{Experimental Section}

Typical procedure for the synthesis of 5-acetoxyuracil (2a). To a stirred mixture of $\mathbf{1 a}(112 \mathrm{mg}, 1.0 \mathrm{mmol})$ and $\mathrm{Pd}(\mathrm{OAc})_{2}$ $(22 \mathrm{mg}, 0.1 \mathrm{mmol})$ in $\mathrm{AcOH}(5 \mathrm{~mL})$ was added $\mathrm{PhI}(\mathrm{OAc})_{2}$ (805 mg, $2.5 \mathrm{mmol}$ ) and heated to $60{ }^{\circ} \mathrm{C}$ for $3 \mathrm{~h}$. After removal of solvent, the residue was purified by column chromatography $\left(\mathrm{CHCl}_{3} / \mathrm{MeOH}, 10: 1\right)$ to afford $\mathbf{2 a}(123 \mathrm{mg}, 72 \%)$ as a white solid. Other compounds were prepared similarly and the spectroscopic data of $\mathbf{2} \mathbf{a}-\mathbf{h}, \mathbf{3 e}$, and $\mathbf{3 f}$ are as follows.

Compound 2a: 72\%; white solid, mp 232 - $234{ }^{\circ} \mathrm{C}$ (decomp.); IR (KBr) 3194, 1781, 1763, $1709 \mathrm{~cm}^{-1} ;{ }^{1} \mathrm{H}$ NMR (DMSO- $d_{6}$, $300 \mathrm{MHz}) \delta 2.19(\mathrm{~s}, 3 \mathrm{H}), 7.55(\mathrm{~s}, 1 \mathrm{H}), 10.88(\mathrm{br} \mathrm{s}, \mathrm{NH}), 11.42$ (br s, NH); ${ }^{13} \mathrm{C}$ NMR (DMSO-d $\left.6,75 \mathrm{MHz}\right) \delta 20.06,125.92$, 133.33, 150.52, 159.26, 168.55; ESIMS $m / z 193\left(\mathrm{M}^{+}+\mathrm{Na}\right)$. Anal. Calcd For $\mathrm{C}_{6} \mathrm{H}_{6} \mathrm{~N}_{2} \mathrm{O}_{4}$ : C, 42.36; H, 3.55; N, 16.47. Found: $\mathrm{C}$, 42.47; H, 3.74; N, 16.21 .

Compound 2b: $60 \%$; white solid, mp $232-234{ }^{\circ} \mathrm{C}$ (decomp.); IR (KBr) 3194, 1781, 1763, $1709 \mathrm{~cm}^{-1} ;{ }^{1} \mathrm{H}$ NMR (DMSO- $d_{6}$, $300 \mathrm{MHz}) \delta 1.95$ (s, 3H), 2.22 (s, 3H), 10.94 (br s, NH), 11.28 (br s, NH); ${ }^{13} \mathrm{C}$ NMR (DMSO- $\left.d_{6}, 75 \mathrm{MHz}\right) \delta 13.08,19.96$, 123.03, 143.38, 150.06, 158.85, 168.40; ESIMS m/z $207\left(\mathrm{M}^{+}+\right.$ $\mathrm{Na}$ ). Anal. Calcd For $\mathrm{C}_{7} \mathrm{H}_{8} \mathrm{~N}_{2} \mathrm{O}_{4}$ : C, 45.66; H, 4.38; N, 15.21 . Found: C, 45.86; H, 4.42; N, 15.03 .

Compound 2c: $56 \%$; white solid, mp $150-152{ }^{\circ} \mathrm{C}$; IR (KBr) 1772, 1709, 1677, 1652, $1208 \mathrm{~cm}^{-1} ;{ }^{1} \mathrm{H} \mathrm{NMR}\left(\mathrm{CDCl}_{3}, 300\right.$ $\mathrm{MHz}) \delta 2.30$ (s, 3H), 3.37 (s, 3H), 3.41 (s, 3H), 7.19 (s, 1H); ${ }^{13} \mathrm{C}$ NMR $\left(\mathrm{CDCl}_{3}, 75 \mathrm{MHz}\right) \delta 20.18,28.27,37.03,126.23$, 134.39, 150.67, 158.57, 168.68; ESIMS $m / z 221\left(\mathrm{M}^{+}+\mathrm{Na}\right)$.

Compound 2d: $71 \%$; white solid, mp $146-148{ }^{\circ} \mathrm{C}$; IR (KBr) $1771,1706,1647,1201 \mathrm{~cm}^{-1} ;{ }^{1} \mathrm{H} \mathrm{NMR}\left(\mathrm{CDCl}_{3}, 300 \mathrm{MHz}\right) \delta$ $2.19(\mathrm{~s}, 3 \mathrm{H}), 2.33(\mathrm{~s}, 3 \mathrm{H}), 3.36(\mathrm{~s}, 3 \mathrm{H}), 3.43(\mathrm{~s}, 3 \mathrm{H}) ;{ }^{13} \mathrm{C} \mathrm{NMR}$ $\left(\mathrm{CDCl}_{3}, 75 \mathrm{MHz}\right) \delta 13.53,20.15,28.34,31.95,124.60,142.99$, 151.12, 157.71, 168.66; ESIMS $m / z 235\left(\mathrm{M}^{+}+\mathrm{Na}\right)$.

Compound 2e: $33 \%$; white solid, mp $128-130{ }^{\circ} \mathrm{C}$; IR (KBr) $1778,1716,1675,1655 \mathrm{~cm}^{-1} ;{ }^{1} \mathrm{H}$ NMR $\left(\mathrm{CDCl}_{3}, 300 \mathrm{MHz}\right) \delta$ $2.29(\mathrm{~s}, 3 \mathrm{H}), 5.03(\mathrm{~s}, 2 \mathrm{H}), 5.21(\mathrm{~s}, 2 \mathrm{H}), 7.28(\mathrm{~s}, 1 \mathrm{H}), 7.47(\mathrm{~d}$, $J=9.0 \mathrm{~Hz}, 2 \mathrm{H}), 7.61(\mathrm{~d}, J=9.0 \mathrm{~Hz}, 2 \mathrm{H}), 8.14(\mathrm{~d}, J=9.0 \mathrm{~Hz}$, $2 \mathrm{H}), 8.22(\mathrm{~d}, J=9.0 \mathrm{~Hz}, 2 \mathrm{H}) ;{ }^{13} \mathrm{C} \mathrm{NMR}\left(\mathrm{CDCl}_{3}, 75 \mathrm{MHz}\right) \delta$ 20.19, 44.60, 52.14, 123.71, 124.34, 127.21, 128.65, 129.85, 133.51, 141.70, 143.02, 147.51, 147.99, 150.35, 158.12, 168.44; ESIMS $m / z 462\left(\mathrm{M}^{+}+\mathrm{Na}\right)$. Anal. Calcd For $\mathrm{C}_{20} \mathrm{H}_{16} \mathrm{~N}_{4} \mathrm{O}_{8}: \mathrm{C}$, 54.55; H, 3.66; N, 12.72. Found: C, 54.79; H, 3.62; N, 12.56 .

Compound 3e: $23 \%$; white solid, mp $172-174{ }^{\circ} \mathrm{C}$; IR (KBr) $1779,1735,1521,1344 \mathrm{~cm}^{-1} ;{ }^{1} \mathrm{H}$ NMR $\left(\mathrm{CDCl}_{3}, 300 \mathrm{MHz}\right) \delta$ $1.70(\mathrm{~s}, 3 \mathrm{H}), 1.98(\mathrm{~s}, 3 \mathrm{H}), 2.03(\mathrm{~s}, 3 \mathrm{H}), 4.59(\mathrm{~d}, J=16.2 \mathrm{~Hz}$, $1 \mathrm{H}), 4.83(\mathrm{~d}, J=15.0 \mathrm{~Hz}, 1 \mathrm{H}), 4.89(\mathrm{~d}, J=15.0 \mathrm{~Hz}, 1 \mathrm{H}), 4.95$ $(\mathrm{d}, J=16.2 \mathrm{~Hz}, 1 \mathrm{H}), 7.19(\mathrm{~s}, 1 \mathrm{H}), 7.47(\mathrm{~d}, J=8.7 \mathrm{~Hz}, 2 \mathrm{H})$, $7.60(\mathrm{~d}, J=8.7 \mathrm{~Hz}, 2 \mathrm{H}), 8.20(\mathrm{~d}, J=8.7 \mathrm{~Hz}, 2 \mathrm{H}), 8.22(\mathrm{~d}, J=$ $8.7 \mathrm{~Hz}, 2 \mathrm{H}) ;{ }^{13} \mathrm{C} \mathrm{NMR}\left(\mathrm{CDCl}_{3}, 75 \mathrm{MHz}\right) \delta 19.85,20.28,20.40$, 42.26, 43.32, 85.10, 85.41, 123.85, 123.89, 128.85, 129.40, $142.04,143.32$, 147.58, 147.75, 155.71, 165.87, 166.77, 167.20, 167.84; ESIMS $m / z 581\left(\mathrm{M}^{+}+\mathrm{Na}\right)$.

Compound 2f: $23 \%$; white solid, mp $56-58{ }^{\circ} \mathrm{C}$; IR (KBr) $1778,1731,1713,1674,1651,1613 \mathrm{~cm}^{-1} ;{ }^{1} \mathrm{H} \mathrm{NMR}\left(\mathrm{CDCl}_{3}\right.$,
$300 \mathrm{MHz}) \delta 2.25(\mathrm{~s}, 3 \mathrm{H}), 3.77(\mathrm{~s}, 3 \mathrm{H}), 3.80(\mathrm{~s}, 3 \mathrm{H}), 4.84(\mathrm{~s}$, $2 \mathrm{H}), 5.08(\mathrm{~s}, 2 \mathrm{H}), 6.83(\mathrm{~d}, J=8.7 \mathrm{~Hz}, 2 \mathrm{H}), 6.89(\mathrm{~d}, J=8.7 \mathrm{~Hz}$, $2 \mathrm{H}), 7.08(\mathrm{~s}, 1 \mathrm{H}), 7.21(\mathrm{~d}, J=8.7 \mathrm{~Hz}, 2 \mathrm{H}), 7.45(\mathrm{~d}, J=8.7 \mathrm{~Hz}$, $2 \mathrm{H}) ;{ }^{13} \mathrm{C} \mathrm{NMR}\left(\mathrm{CDCl}_{3}, 75 \mathrm{MHz}\right) \delta 20.26,44.68,51.89,55.20$, 55.30, 113.74, 114.57, 126.47, 126.77, 128.56, 129.94, 130.79, $132.88,150.62,158.40,159.17,159.87,168.58 ;$ ESIMS $m / z$ $433\left(\mathrm{M}^{+}+\mathrm{Na}\right)$.

Compound 3f: $21 \%$; white solid, mp $48-50{ }^{\circ} \mathrm{C}$; IR (KBr) $1770,1731,1613 \mathrm{~cm}^{-1} ;{ }^{1} \mathrm{H} \mathrm{NMR}\left(\mathrm{CDCl}_{3}, 300 \mathrm{MHz}\right) \delta 1.53(\mathrm{~s}$, $3 \mathrm{H}), 1.85(\mathrm{~s}, 3 \mathrm{H}), 2.00(\mathrm{~s}, 3 \mathrm{H}), 3.78(\mathrm{~s}, 6 \mathrm{H}), 4.34(\mathrm{~d}, J=15.6$ $\mathrm{Hz}, 1 \mathrm{H}), 4.69$ (s, 2H), 4.97 (d, $J=15.6 \mathrm{~Hz}, 1 \mathrm{H}), 6.83$ (d, $J=$ $8.7 \mathrm{~Hz}, 2 \mathrm{H}), 6.84(\mathrm{~d}, J=8.7 \mathrm{~Hz}, 2 \mathrm{H}), 7.16(\mathrm{~s}, 1 \mathrm{H}), 7.21(\mathrm{~d}, J=$ $8.7 \mathrm{~Hz}, 2 \mathrm{H}), 7.36(\mathrm{~d}, J=8.7 \mathrm{~Hz}, 2 \mathrm{H}) ;{ }^{13} \mathrm{C} \mathrm{NMR}\left(\mathrm{CDCl}_{3}, 75\right.$ MHz) $\delta 19.73,20.14,20.39,42.22,43.27,55.25,55.30,84.94$, $85.51,113.93,113.96,127.98,128.35,129.76,130.06,155.95$, $159.21,159.29,166.33,167.06,167.57,167.71$; ESIMS $m / z$ $551\left(\mathrm{M}^{+}+\mathrm{Na}\right)$.

Compound 2g: 55\%; white solid, mp 77 - $79{ }^{\circ} \mathrm{C}$; IR (KBr) $3230,1748,1715,1231 \mathrm{~cm}^{-1} ;{ }^{1} \mathrm{H} \mathrm{NMR}\left(\mathrm{CDCl}_{3}, 300 \mathrm{MHz}\right) \delta$ $2.11(\mathrm{~s}, 3 \mathrm{H}), 2.13(\mathrm{~s}, 3 \mathrm{H}), 2.14(\mathrm{~s}, 3 \mathrm{H}), 2.29(\mathrm{~s}, 3 \mathrm{H}), 4.30-4.41$ $(\mathrm{m}, 3 \mathrm{H}), 5.31-5.33(\mathrm{~m}, 2 \mathrm{H}), 6.06-6.11(\mathrm{~m}, 1 \mathrm{H}), 7.51(\mathrm{~s}, 1 \mathrm{H})$, 9.25 (br s, NH); ${ }^{13} \mathrm{C} \mathrm{NMR}\left(\mathrm{CDCl}_{3}, 75 \mathrm{MHz}\right) \delta 20.22,20.36$, 20.47, 20.61, 63.01, 70.06, 72.90, 80.08, 87.23, 127.94, 130.24, 149.21, 157.74, 168.17, 169.54, 169.61, 170.08; ESIMS $m / z$ $451\left(\mathrm{M}^{+}+\mathrm{Na}\right)$. Anal. Calcd For $\mathrm{C}_{17} \mathrm{H}_{20} \mathrm{~N}_{2} \mathrm{O}_{11}$ : C, 47.67; H, 4.71; N, 6.54. Found: C, 47.94; H, 4.94; N, 6.29.

Compound 2h: $25 \%$; white solid, mp $106-108^{\circ} \mathrm{C}$; IR (KBr) $3188,1781,1723,1697,1660 \mathrm{~cm}^{-1}$; ${ }^{1} \mathrm{H} \mathrm{NMR}\left(\mathrm{CDCl}_{3}, 300 \mathrm{MHz}\right)$ $\delta 0.07(\mathrm{~s}, 3 \mathrm{H}), 0.08(\mathrm{~s}, 3 \mathrm{H}), 0.10(\mathrm{~s}, 6 \mathrm{H}), 0.89(\mathrm{~s}, 9 \mathrm{H}), 0.92(\mathrm{~s}$, $9 \mathrm{H}), 2.00-2.09(\mathrm{~m}, 1 \mathrm{H}), 2.26(\mathrm{~s}, 3 \mathrm{H}), 2.26-2.35(\mathrm{~m}, 1 \mathrm{H}), 3.75$ (dd, $J=11.4$ and $2.1 \mathrm{~Hz}, 1 \mathrm{H}), 3.88-3.95(\mathrm{~m}, 2 \mathrm{H}), 4.37-4.41$ $(\mathrm{m}, 1 \mathrm{H}), 6.31(\mathrm{t}, J=6.3 \mathrm{~Hz}, 1 \mathrm{H}), 7.82(\mathrm{~s}, 1 \mathrm{H}), 8.89$ (br s, $\mathrm{NH}) ;{ }^{13} \mathrm{C} \mathrm{NMR}\left(\mathrm{CDCl}_{3}, 75 \mathrm{MHz}\right) \delta-5.59,-4.88,-4.64,17.97$, $18.39,20.19,25.71,25.88,41.80,62.79,71.79,85.46,88.01$, 127.40, 131.13, 149.16, 158.04, 168.28; ESIMS $\mathrm{m} / \mathrm{z} 537$ $\left(\mathrm{M}^{+}+\mathrm{Na}\right)$. Anal. Calcd For $\mathrm{C}_{23} \mathrm{H}_{42} \mathrm{~N}_{2} \mathrm{O}_{7} \mathrm{Si}_{2}: \mathrm{C}, 53.67 ; \mathrm{H}, 8.22$; N, 5.44. Found: C, 53.82; H, 7.98; N, 5.33.

Acknowledgments. This research was supported by Basic Science Research Program through the National Research Foundation of Korea (NRF) funded by the Ministry of Education, Science and Technology (2009-0070633). Spectroscopic data was obtained from the Korea Basic Science Institute, Gwangju branch.

\section{References and Notes}

1. For the electrophilic palladation of various aromatic compounds including indoles, see: (a) Stuart, D. R.; Fagnou, K. Science 2007, 316, 1172-1175. (b) Li, R.; Jiang, L.; Lu, W. Organometallics 2006, 25, 5973-5975. (c) Deprez, N. R.; Kalyani, D.; Krause, A.; Sanford, M. S. J. Am. Chem. Soc. 2006, 128, 4972-4973. (d) Daugulis, O.; Zaitsev, V. G. Angew. Chem. Int. Ed. 2005, 44, 40464048. (e) Cheng, D.; Gallagher, T. Org. Lett. 2009, 11, 2639-2641. (f) Bravo, J.; Cativiela, C.; Navarro, R.; Urriolabeitia, E. P. J. Organometal. Chem. 2002, 650, 157-172. (g) Park, J.-W.; Jun, C.-H. ChemCatChem. 2009, 1, 69-71.

2. For the Pd-catalyzed acetoxylations, see: (a) Desai, L. V.; Stowers, K. J.; Sanford, M. S. J. Am. Chem. Soc. 2008, 130, 13285-13293. 
(b) Kalyani, D.; Sanford, M. S. Org. Lett. 2005, 7, 4149-4152. (c) Dick, A. R.; Kampf, J. W.; Sanford, M. S. Organometallics 2005, 24, 482-485. (d) Dick, A. R.; Hull, K. L.; Sanford, M. S. J. Am. Chem. Soc. 2004, 126, 2300-2301. (e) Desai, L. V.; Malik, H. A.; Sanford, M. S. Org. Lett. 2006, 8, 1141-1144. (f) Tang, S.; Peng, P.; Wang, Z.-Q.; Tang, B.-X.; Deng, C.-L.; Li, J.-H.; Zhong, P.; Wang, N.-X. Org. Lett. 2008, 10, 1875-1878. (g) Wang, G.-W.; Yuan, T.-T.; Wu, X.-L. J. Org. Chem. 2008, 73, 4717-4720. (h) Kim, S. H.; Lee, H. S.; Kim, S. H.; Kim, J. N. Tetrahedron Lett. 2008, 49, 5863-5866.

3. For the Pd-catalyzed acetoxylation of indole, see: Mutule, I.; Suna, E.; Olofsson, K.; Pelcman, B. J. Org. Chem. 2009, 74, 71957198.

4. For the 5-hydroxyuracil derivatives as oxidative lesions of DNA, see: (a) Riviere, J.; Bergeron, F.; Tremblay, S.; Gasparutto, D.; Cadet, J.; Wagner, J. R. J. Am. Chem. Soc. 2004, 126, 6548-6549. (b) Kang, J. I., Jr.; Sowers, L. C. Chem. Res. Toxicol. 2008, 21, 1211-1218. (c) Rabi, J. A.; Fox, J. J. J. Org. Chem. 1972, 37, 38983901. (d) Thiviyanathan, V.; Somasunderam, A.; Volk, D. E.; Hazra, T. K.; Mitra, S.; Gorenstein, D. G. Biochem. Biophy. Res. Commun. 2008, 366, 752-757.

5. For the synthesis and applications of 5-hydroxy- and 5-acetoxyuracil derivatives, see: (a) Kondo, Y.; Witkop, B. J. Am. Chem. Soc. 1969, 91, 5264-5270. (b) Majumdar, K. C.; Chattopadhyay, B. Synlett 2008, 979-982. (c) Grabovskiy, S. A.; Abdrakhmanova, A. R.; Murinov, Y. I.; Kabal'nova, N. N. Curr. Org. Chem. 2009, 13, 1733-1736. (d) Majumdar, K. C.; Mondal, S. Tetrahedron 2009, 65, 9604-9608.

6. For the examples of Pd-catalyzed functionalization of uracil moiety at the 5-position, see: (a) Bergstrom, D. E.; Ogawa, M. K. J. Am. Chem. Soc. 1978, 100, 8106-8112. (b) Bigge, C. F.; Kalaritis, P.; Deck, J. R.; Mertes, M. P. J. Am. Chem. Soc. 1980, 102, 20332038. (c) Ruth, J. L.; Bergstrom, D. E. J. Org. Chem. 1978, 43, 2870-2876. (d) Mendonca, F. J. B., Jr.; dos Anjos, J. V.; Sinou, D.; de Melo, S. J.; Srivastava, R. M. Synthesis 2007, 1890-1897. (e) Amblard, F.; Nolan, S. P.; Schinazi, R. F.; Agrofoglio, L. A. Tetrahedron 2005, 61, 537-544.
7. For our recent papers on palladium-catalyzed reactions, see: (a) Gowrisankar, S.; Lee, H. S.; Kim, S. H.; Lee, K. Y.; Kim, J. N. Tetrahedron 2009, 65, 8769-8780. (b) Kim, H. S.; Gowrisankar, S.; Kim, S. H.; Kim, J. N. Tetrahedron Lett. 2008, 49, 3858-3861. (c) Gowrisankar, S.; Lee, H. S.; Lee, K. Y.; Lee, J.-E.; Kim, J. N. Tetrahedron Lett. 2007, 48, 8619-8622. (d) Kim, H. S.; Lee, H. S.; Kim, S. H.; Kim, J. N. Tetrahedron Lett. 2009, 50, 3154-3157. (e) Kim, H. S.; Gowrisankar, S.; Kim, E. S.; Kim, J. N. Tetrahedron Lett. 2008, 49, 6569-6572. (f) Gowrisankar, S.; Kim, S. H.; Kim, J. N. Bull. Korean Chem. Soc. 2009, 30, 726-728. (g) Gowrisankar, S.; Kim, K. H.; Kim, J. N. Bull. Korean Chem. Soc. 2008, 29, 2537-2539.

8. Although the reaction of $1 \mathrm{a}$ and PIDA at $60^{\circ} \mathrm{C}$ without a palladium catalyst did not produce any trace amounts of $\mathbf{2 a}$, we obtained 2a in $59 \%$ when we run the reaction at $100{ }^{\circ} \mathrm{C}$ with only PIDA $(2.5$ equiv) even in the absence of a palladium catalyst. Thus, there might be acting another mechanism of acetoxylation which is possible with PIDA only. Scrutinizing of reported papers involving the use of PIDA revealed that electron-rich aromatic compounds ${ }^{10}$ and enamino esters ${ }^{11}$ could be acetoxylated. However, the reactions of $\mathbf{1 e - h}$ and PIDA were very sluggish in the absence of a palladium catalyst even at $100^{\circ} \mathrm{C}$, and the results stated that these compounds are not sufficiently nucleophilic to be acetoxylated with PIDA.

9. Cleavage of $\mathrm{N}$-glycoside bond as well as formation of the corresponding iodonium salt might be the reason of low yield. For the formation of iodonium salt, see: (a) Campbell, J. A.; Broka, C. A.; Gong, L.; Walker, K. A. M.; Wang, J.-H. Tetrahedron Lett. 2004, 45, 4073-4075. (b) Roh, K. R.; Kim, J. Y.; Kim, Y. H. Chem. Lett. 1998, 1095-1096.

10. For the examples of direct acetoxylation of electron-rich aromatic compounds with PIDA, see: (a) Barlin, G. B.; Riggs, N. V. J. Chem. Soc. 1954, 3125-3128. (b) Prakash, O.; Kaur, H.; Sharma, V.; Bhardwaj, V.; Pundeer, R. Tetrahedron Lett. 2004, 45, 9065-9067. (c) Quideau, S.; Pouysegu, L.; Avellan, A.-V.; Whelligan, D. K.; Looney, M. A. Tetrahedron Lett. 2001, 42, 7393-7396.

11. For the example of direct acetoxylation of enamine esters with PIDA, see: Wang, J.-Y.; Liu, S.-P.; Yu, W. Synlett 2009, 2529-2533. 